It is clear that if you want to have a commercially viable biotechnology company, you must use your resources to go after major diseases that aren't treated very effectively today.

\section{-George Scangos \\ President and CEO, Exelixis Pharmaceuticals, South San Francisco, CA}

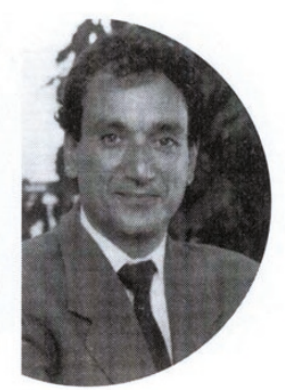

drug discovery process. The selection of the target, if done properly, will have a significant impact on the treatment of human diseases and lead to commercial success. However, the parameters that we need for good decision-making for selection of these drug targets has not yet been fully developed.

Genomics research has given us information and tools. We have sequence information from the human genome and other organisms that are of value; tools coming from positional cloning have been useful for constructing maps, although have yet to deliver on the more complex human dis- eases. Much of the work that has been done in genomics has led to the creation of a physical map; we have sequences, landmarks on the genome; guideposts for positional cloning, and candidate genes to guide pharmacogenetic efforts, but there is a lot more to be done here.

The necessary next step is to overlay on the physical map a functional map whereby additional information is tied to the gene sequence information. The sequence information is not sufficient in itself. We need to have the overlay of functional information that includes many things. It includes the ability to predict the function of a gene based on its sequence; its similarities to other gene sequences; the ability to predict from its structure the type of molecules that interact or bind to it; and importantly, the type of biological activity that is linked to the particular gene sequence or gene product.

This functional map needs to be constructed and tied in strongly with an informatics component. Informatics will drive not only the ability to analyze and manipulate the data, but the entire decision-making process. What we envision here is having our chemistry databases, our sequence, our structural information, all tied together with biological information, and information on gene function and pathways.

Efforts from developmental biology that we have heard at this meeting are very important and will solidly contribute to our understanding of this biological function. It needs to be tied in with the other elements required for drug discovery.

In this way, we can imagine having informatics drive a better decision-making process for target selection. We would be able to weigh, for instance, whether the target is

\title{
In a fly's eye
}

How can the development of the compound eye in flies be related to the genesis of tumors in humans? "Many of the components of the core biological processes of developmental and physiological functions are well conserved between invertebrates and humans," explains Gerald Rubin, Howard Hughes Medical Institute (HHMI) investigator from the University of California, Berkeley. Rubin's work focuses on studying assembling genes in developmental pathways by using Drosophila genetics. It just so happens that Drosophila eye development is mediated by Rasl, a homologue of mammalian oncogenes $\mathrm{H}$-ras, $\mathrm{K}$-ras, and $\mathrm{N}$-ras, a well-studied pathway for a variety of human cancers.

Rubin's use of Drosophila eye phenotypes as a screen for probing Ras-pathway-based differentiation seemed to naturally lend itself as a model system for understanding the role and function of human Ras homologs in cancer. While Rubin admits that humans have potentially a much greater number of genes that could participate in this pathway upstream, he thinks the beauty of studying Drosophila is that you get a much higher sig-

Robert E. Welikson is at the department of cell biology and anatomy, Cornell University Medical School, 1300 York Ave., New York, NY 10021 nal to noise ratio. "Although the human genome contains 70,000 genes, these genes will encode the components of only a few hundred multicomponent processes,"says Rubin.

Leonard Zon, HHMI investigator at Children's Hospital, Boston, Mass., thinks that developmental models for diseases such as cancer are likely to yield unique insights that may be more difficult to tease out in adult models. "Cancer can be viewed as a recapitulation of developmental programs gone awry," he says. Zon's own work focuses on understanding hematopoiesis and the development of the immune system using zebrafish as a model system. Using mutants defective in blood formation, he has characterized 80 genes that have mammalian homologues in normal hematopoiesis.

Zon describes the basis of his approach as "genomic ping-ponging." Since the zebrafish and human genomes are syntenic, this allows him to use candidate genes produced from genetic screens of zebrafish to search human genomic databases to identify their human homologues. Once these human homologues are identified, drug design can proceed in a more logical manner. Alternatively, genes linked to human diseases can be used to identify homologues in zebrafish, an organism in which its developmental significance can be readily tested.
But model systems in this brave new world of therapeutic development need not be limited to flies and fish. Worms too, have a role. Gary Ruvkun (Harvard Medical School, Boston, MA) presented data that demonstrated that studies in the metabolism and longevity of the nematode C. elegans may be used to understand and treat diabetes. Ruvkun's group has found a link between the C. elegans' daf-16 gene, which codes for a family of Forkhead transcription factors and shows homology to human genes that transduce insulin or insulin-like growth factors signals, and diabetes. The loss of daf-16 obviates the need for insulin signaling, proving itself as a major checkpoint of insulin-like signaling pathway. "In fact, daf-16 turns out to be the only target that suppressed the metabolic disorder caused by insulin-like defects in C. elegans," says Ruvkun. He suggests that this makes its human homologues key targets for drug development.

So, will developmental biology be the postgenomic stepping stone to better drugs? "Although the field is fast maturing" says Zon, "we are still in the black box phase." Zon and leaders in the field have little doubt that developmental biology, will yield dramatic insights into important genetic pathways. But how long it will take these revelations to yield cost-effective drugs is still anyone's guess.

Robert E. Welikson 